
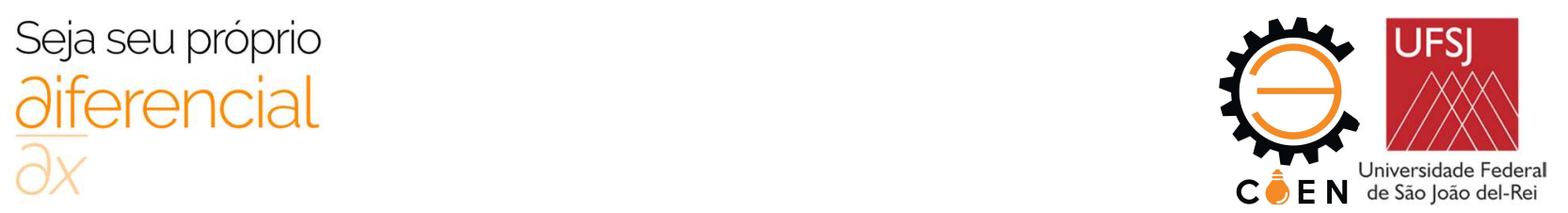

\title{
IMPRESSÃO 3D: UM GUIA PRÁTICO
}

Pedro Coelho Silva ${ }^{(1)}$ (ocoelhopedro@gmail.com), Rafael Souza Santandrea ${ }^{(1)}$ (r.santandrea97@gmail.com ), Victor Lattaro Volpini ${ }^{(1)}$ (victorlattaro@gmail.com), Lincoln Cardoso Brandão ${ }^{(1)}$ (lincoln@ufsj.edu.br)

(1) Universidade Federal de São João del-Rei (UFSJ) - Engenharia Mecânica - Praça Frei Orlando, nº 170 - Centro - São João delRei-MG

RESUMO: AImpressão3D é uma tecnologia versátil e com potencial de trazer grandes mudanças no modo que fabricamos os produtos. Também chamada de Manufatura aditiva, teve as principais metodologias criadas na década de 80, porém, somente após o vencimento das principais patentes, ocorreu sua popularização. Sua operação ainda é considerada complexa para os usuários iniciantes, principalmente devido à falta de documentação para orientação no uso. Ao aplicar uma metodologia com o objetivo de identificar na literatura e na experiência prática dos autores os procedimentos necessários para se utilizar uma impressora desse tipo, o presente artigo apresenta os resultados de maneira simples e objetiva, gerando um guia prático para os novos usuários. Assim serão apresentadas recomendações para a aquisição de uma impressora: os programas necessários para sua operação; as configurações disponíveis e o como interferem na qualidade final dos produtos; como evitar erros de impressão e o que é necessário para dar acabamento aos mesmos. Na conclusão, em uma lista objetiva, será comentado cada um dos tópicos, acrescentando recomendações dos autores.

PALAVRAS-CHAVE: impressão 3D, guia, manufatura aditiva, fabricação aditiva

\section{INTRODUÇÃO}

Uma tecnologia emergente com alto potencial de trazer mudanças significativas à sociedade é a impressão tridimensional(AGUIAR, 2016). Reduzido como Impressão 3D, do inglês 3D Printing (3DP), originalmente, se tratava de uma das metodologias de fabricação englobadas nas Manufaturas Aditivas (AM,Additive Manufacturing ), ou fabricação aditiva, mas passou a assumir um sentido de sinônimo da mesma. AM de fineto do processo de fabricação por deposição ou união de material em camadas formando uma geometria tridimensional complexa, sem a necessidade de processos adicionais. Por seus custos de fabricação serem praticamente lineares, ou seja, o custo para se produzir qualquer quantidade de produtos se mantém linearmente proporcional, se destacou como um dos principais processos de Prototipagem Rápida (Rapid Prototyping), mas com sua evolução se mostrou prática para fabricação de mais variados produtos (MARQUARDT \& ZHENG, 2016).

Com as principais patentes na década de 80 , por vinte anos, enquanto a tecnologia estava protegida, AM se limitava a equipamentos industriais, mas com a expiração das patentes, e a entrada de concorrentes e equipamentos de pequeno porte, permitiu o barateamento do equipamento e sua popularização (MARQUARDT \& ZHENG, 2016). Assim a 3DP já é aplicada comercialmente em produtos ergonômicos e equipamentos especiais para a medicina (CULMONE et al., 2019), fabricação de produtos personalizados, ferramentas para fabricação tradicional, peças de reposição e manutenção, e até na construção civil (BERMAN, 2012).

Para operar uma 3DP é necessário construir um modelo virtual do objeto que se pretende fabricar, utilizando um programa de modelagem CAD (Design Auxiliado por Computador; Computer Aided Design). Após esse processo é necessário interpretar as geometrias criadas e criar o "caminho da ferramenta", definindo os procedimentos de operação da máquina que será utilizada para atingir

IX COEN - Congresso de Engenharias da UFSJ Interconexão. 

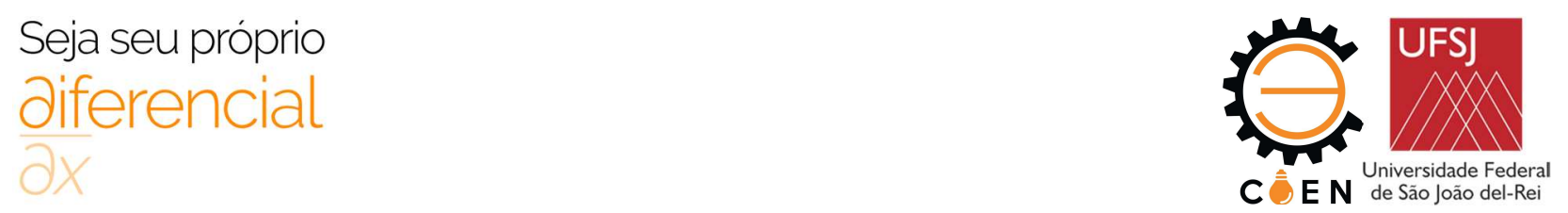

os fins, esse processo é chamado de Fatiar (Slicing, tradução nossa). Em seguida deve-se preparar a máquina, calibrar, colocar os materiais necessários e transmitir o código gerado para a máquina, que irá operar e gerar o sólido automaticamente. Por fim é necessário retirar o produto criado da plataforma de impressão; remover os suportes e qualquer excesso de material; reaquecer ou curar o material se for preciso aumentar a resistência; e fazer o acabamento das superfícies, montagem e pintura quando necessário (HIEMENZ, 2011).

Em suas investigações, Aguiar (2016) destacou a ausência de um método prático para orientar a utilização da 3DP, sendo necessário uma extensa investigação bibliográfica, além da disponibilidade de tempo para explorar e aprender os percalços no processo. Assim é necessário criar um material claro para facilitar a entrada da tecnologia em espaços mais diversos, além de combater os ceticismos e medos envolvendo as novas tecnologias.

\section{OBJETIVO}

A presente pesquisa aplicada tem o objetivo de identificar na literatura os procedimentos recomendados para se operar uma Impressora 3D.

\section{METODOLOGIA}

A "metodologia científica", segundo Kuark (2010), é uma coleção de pressupostos para realizar e apresentar um trabalho de pesquisa, visando a eficácia deste e dos resultados, proporcionando um padrão reconhecível por outros pesquisadores e público geral. Dentre as formas de se expor os resultados é possível gerar um guia, um obra que apresenta de modo simples e objetivo os resultados, construindo uma ferramenta útil e de fácil manuseio.

A presente investigação buscou na literatura técnica e científica materiais que apresentam a metodologia de fabricação estudada; sua forma de operação; suas vantagens e desvantagens; erros comuns e suas soluções. Com este material, e a experiência prática na operação dos equipamentos pelos autores, foi desenvolvido um guia de operação para uma Impressora 3D.

\section{IMPRESSÃO TRIDIMENSIONAL}

A Impressão 3D, ou Manufatura Aditiva, define todo processo de fabricação em que, ao contrário da manufatura subtrativa, constrói o produto final a partir de um material base pela adição contínua do material em camadas, formando o sólido final (AGUIAR, 2016).

Ao contrário de processos de fabricação em massa, como a fabricação por injeção em moldes, a 3DP apresenta menor custo fixo, ou seja, por não ser necessário fabricar as ferramentas necessárias para a fabricação de um produto específico. Em comparação com processos de fabricação por subtração de material, como a usinagem, a 3DP apresenta uma operação com menor quantidade de resíduos, ou seja, além de se obter um objeto mais próximo que o pretendido em um processo único, sendo necessário apenas furação e acabamentos superficiais em alguns casos, reduz em 40\% o desperdício de material para se obter um mesmo formato. Além disso, até $98 \%$ do material removido ou descartado na impressão ou acabamento são recicláveis para a 3DP. Em complemento a impressão permite obter geometrias impossíveis de serem obtidas somente pelos processos subtrativos, como a remoção de material em regiões que sofrem menor esforço. Por outro lado, os custos para produção em massa ainda são altos e, dependendo da impressora adquirida, há limitações quanto aos materiais

IX COEN - Congresso de Engenharias da UFSJ Interconexão. 

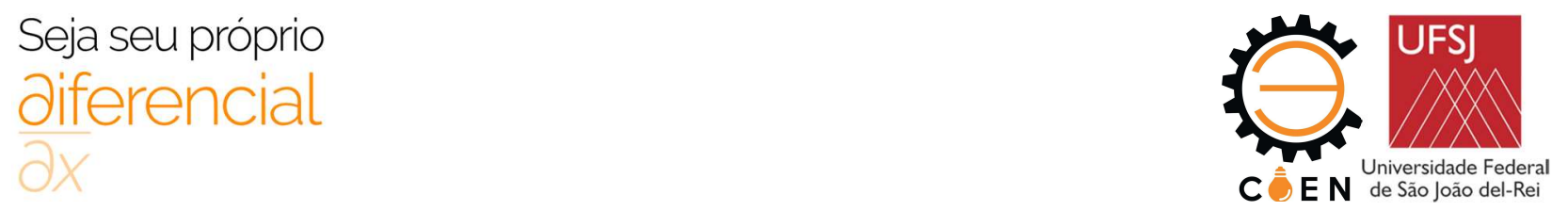

possíveis e suas resistências físicas (HIEMENZ, 2011; BERMAN, 2012; FERNANDEZ-VICENTE et al., 2015; CULMONE et al., 2019).

Os tipos de 3DP e suas siglas são definidas pela norma "ASTM 52900-15 - Terminologia padrão para manufatura aditiva", conforme Culmone e colaboradores (2019). Fizemos a tradução listada abaixo:

- $\quad$ Jateamento de aglomerante (BJ, Binder Jetting);

- $\quad$ Deposição direta por aplicação de energia (DED, Direct Energy Deposition);

- $\quad$ Extrusão de material (ME, Material Extrusion);

- $\quad$ Jateamento de material (MJ, Material Jetting);

- $\quad$ Fusão seletiva de material em pó (PBF, Powder Bad Fusion);

- $\quad$ Laminação de chapas (SL, Sheet Lamination);

- $\quad$ Fotopolimerização de líquido (VP, Vat Photopolymerization).

BJ é o processo de fabricação por aplicação seletiva de um líquido aglomerante, como uma cola, a um material em pó. DED é a fabricação cujo processo consiste na aplicação de energia térmica concentrada, como um laser, para fundir o material sólido enquanto é depositado. ME, fabricação por extrusão e deposição de um termoplástico ou solução por meio de um bocal, inclui o modelamento por deposição de material fundido (FDM, fused deposition modeling; ou FFF, fused filament fabrication). MJ, fabricação por deposição seletiva de material líquido, curado por energia luminosa a cada camada. PBF, fabricação em que a energia térmica funde seletivamente regiões em uma "cama" de material em pó, comumente utilizado para materiais metálicos. SL, fabricação por corte, sobreposição e união de chapas de material por fundição ou aplicação de aglomerante, foi criada no século 19 e aprimorada junto da evolução da AM. VP é a fabricação por polimerização de um líquido, e solidificação do mesmo, pela aplicação de laser ou energia luminosa, de um projetor ou tela, inclui a estereolitografia (STL, stereolithography) e o processamento digital de luz (DLP, digital light process), processos comuns para fabricação de equipamentos médicos devido a sua precisão e materiais utilizados (CULMONE et al., 2019).

Visto a complexidade e a variedade de equipamentos, é necessário identificar quais processos são limitados ao ambiente industrial e quais são viáveis para usuários iniciantes. As impressoras baseadas na ME, as FFF ou FDM (termo registrado por Sott Crum em 1989), apresentam maior simplicidade na arquitetura das impressoras e se popularizaram a partir do projeto RepRap (The Replicating Rapid Prototyper; Prototipador Rápido Auto-replicante, tradução nossa), criado por Adrian Bowyer. Esse projeto iniciou suas publicações em 2004 e em 2007 finalizou o primeiro equipamento. $\mathrm{O}$ objetivo foi construir uma 3DP utilizando peças que podem ser produzidas pelo próprio equipamento, junto de componente de fácil aquisição no varejo. Esse processo, devido a sua arquitetura aberta, difundiu conhecimentos para que outras pessoas e empresas construíssem impressoras com custo mais acessível, permitindo uma variedade de pesquisas sobre essa plataforma e a entrada de novas empresas e produtos no mercado (AGUIAR, 2016; CULMONE et al, 2019).

\subsection{Escolhendo a Impressora 3D}

Para se definir qual equipamento deve ser adquirido é necessário definir algumas questões quanto à operação do equipamento, como o nível técnico de operação e instalação da impressora, a qualidade e velocidade de impressão, as conexões, interfaces, compatibilidades, complementos e os custos de aquisição e operação (AGUIAR, 2016).

IX COEN - Congresso de Engenharias da UFSJ Interconexão. 

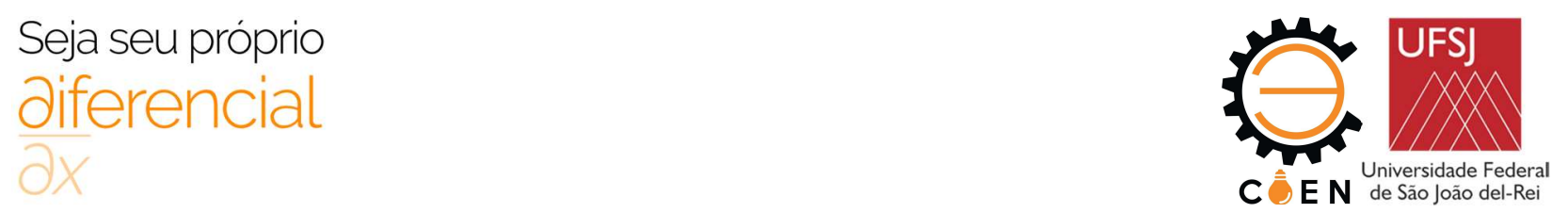

A impressora baseada na RepRap apresenta menor custo e uma comunidade ativa que auxilia no uso. Geralmente é fornecida desmontada e com um sistema mais simples e customizável, no entanto, é de instalação e operação mais complicada (AGUIAR, 2016).

Uma impressora de arquitetura proprietária, como a Cube, fabricada pela 3D System, geralmente é fornecida montada, incluindo os programas e conexões necessárias para a instalação e operação, sendo mais viável para usuários iniciantes, mas a um custo de aquisição de aproximadamente o dobro da outra e de operação quase cinco vezes maior quando comparada com a de arquitetura aberta (AGUIAR, 2016).

Uma terceira alternativa é uma 3DP de arquitetura de operação aberta, mas de projeto de arquitetura fechada que, apesar de apresentar um custo de aquisição próximo ao equipamento da 3D System, apresenta um custo de operação próximo ao da RepRap, sem perder a facilidade de uso similar à da anterior (AGUIAR, 2016).

\subsection{Projeto e Modelo}

Para fazer o projeto é necessário conhecer as propriedades dos materiais disponíveis para serem utilizados na impressora e suas limitações, como as inclinações e espaços vazios máximos nas peças e espessuras mínimas recomendadas pelos fabricantes dos materiais e da impressora, além das dicas disponíveis nas comunidades digitais (FERNANDEZ-VICENTE et al., 2015).

Quanto à resistência do material é possível fazer a otimização da forma, com o objetivo de se obter a forma necessária para se atingir a resistência pretendida. Para a 3DP é comum se utilizar a otimização topológica por simulação, que é um tipo de ferramenta de design que irá fazer o cálculo de otimização da geometria baseado nos requisitos do projeto. Outra alternativa é alterar o preenchimento, que pode ser em estrutura celular, que possibilita atingir uma mesma resistência, com menor massa, densidade e tempo de fabricação (OROPALLO \& PIEGL, 2015).

Os programas CAD disponíveis para modelo podem ser pagos, gratuitos ou de arquitetura aberta, sendo de todos os tipos utilizados na comunidade. Dentre os pagos se destacam o SolidWorks e o Autodesk Inventor, softwares amplamente utilizados para engenharia mecânica, além deles, há o Autodesk Fusion 360 que, apesar de possuir menos recursos, apresenta custo de aquisição menor (BERMAN, 2012). Os programas da Autodesk ainda possuem versão gratuita para a comunidade acadêmica, que atende ao necessário (AUTODESK, 2019). Dentre os gratuitos se destacam o SketchUp e o online Tinkercad, também da Autodesk. Alternativamente, é possível gerar os sólidos por escaneamento tridimensional que utiliza uma câmera e um aplicativo para gerar o sólido (Photogrammetry, Fotogrametria), como o descontinuado 123D Catch, também da Autodesk, o Kinect da Microsoft ou o Sence da 3D System; ou por equipamentos especializados de escaneamento a laser e seus softwares (BERMAN, 2012; AGUIAR, 2016).

Segundo Aguiar (2016), caso a pessoa não tenha conhecimento em modelagem, é possível aproveitar os repositórios, que oferecem muitos modelos gratuitos disponíveis à comunidade:

- $\quad$ Thingiverse - Repositório gratuito, com uma comunidade muito ativa, para diversos tipos de aplicação (MAKERBOT, 2019);

- GrabCAD - Complementar à um programa de modelagem e outro de impressão, o repositório gratuito inclui dicas e tutoriais criados pela comunidade (STRATASYS, 2019);

- MyMiniFactory - Repositório que possui modelos pagos e gratuitos, apresenta a garantia de que todos os objetos na rede são verificados para impressão (MYMINIFACTORY, 2019); 


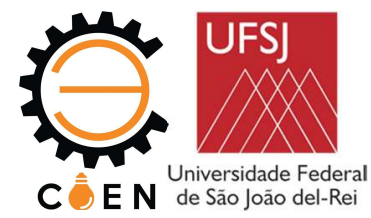

- $\quad$ Repables - Comunidade independente para o compartilhamento aberto de modelos para impressão 3D (REPABLES, 2019).

Após obtido o modelo tridimensional, é necessário fazer sua interpretação para o código que a máquina é capaz de interpretar em sua operação. Caso o programa selecionado não possua um sistema de CAM, que faz a interpretação da geometria e define o caminho da máquina, é necessário exportar para um programa que irá fazer esse processo. Para isso se utiliza um formato de arquivo específico para 3DP, o STL (Stereolithography file format) ou similar (OROPALLO \& PIEGL, 2015). Geralmente o fornecedor da 3DP também providencia o programa para isso, como o Cube Print App oferecido pela 3D System, reduzindo o número de configurações necessárias para se adaptar às especificidades de cada impressora. Caso contrário, também há programas gratuitos, como o MatterControl, criado para preparar um modelo para uma impressora RepRap (AGUIAR, 2016).

Os fatiadores irão gerar automaticamente o código necessário para operar a 3DP, baseado nos parâmetros informados pelo usuário. Os que são fornecidos com as impressoras geralmente são mais simples de se utilizar, dando a liberdade do usuário configurar somente as relações do modelo, como o material utilizado, a densidade do preenchimento, a construção de elementos de apoio para superfícies livres e as superfícies de aderência à mesa de impressão. Como as impressoras de arquitetura aberta variam muito, os programas direcionados às mesmas permitem ao usuário, além das configurações padrão, fazer ajustes em relação à própria impressora, como as velocidades de impressão, temperaturas e ajuste de eixos, permitindo também a correção de alguns erros inerentes ao próprio equipamento (AGUIAR, 2016).

Antes de se dar início à impressão propriamente dita, é necessário verificar se os parâmetros de impressão estão corretos e a impressora devidamente calibrada. Configurações adequadas do fatiador, além de definir a resistência do produto, podem significar a diferença entre o sucesso e o fracasso de uma impressão. Por isso é muito importante saber como os fatiadores funcionam e como cada configuração vai afetar os resultados (ALL3DP, 2016). Os parâmetros de impressão mais comuns são:

- $\quad$ Altura da camada (layer height);

- $\quad$ Espessura da parede (shell thickness);

- $\quad$ Retração (retraction);

- $\quad$ Densidade de preenchimento (infill density);

- $\quad$ Velocidade de impressão (printing speed);

- $\quad$ Suporte (support);

- $\quad$ Tipo de adesão à plataforma (platform adhesion type) (ALL3DP, 2016, tradução nossa).

A altura da camada pode ser considerada como a resolução da sua impressão. Impressões feitas com camadas mais finas resultarão em impressões mais detalhadas com faces mais lisas em que é mais difícil ver as camadas de filamento individuais. Camadas mais finas levam mais tempo para imprimir algo, pois haverá mais camadas no objeto impresso. Camadas mais grossas proporcionam impressões mais rápidas, mas menos detalhadas e com as camadas mais visíveis. Impressões com baixa resolução são boas para prototipagem em que detalhes superficiais podem não ser necessários (ALL3DP, 2016).

A espessura da parede está relacionada ao número de vezes que as linhas externas serão traçadas pela impressora antes de começar a parte vazia da sua peça. Isso define a espessura das paredes laterais e é um dos principais fatores relacionados à resistência da sua impressão. Aumentar esse número cria paredes mais grossas e melhora a resistência da peça. A densidade de preenchimento 

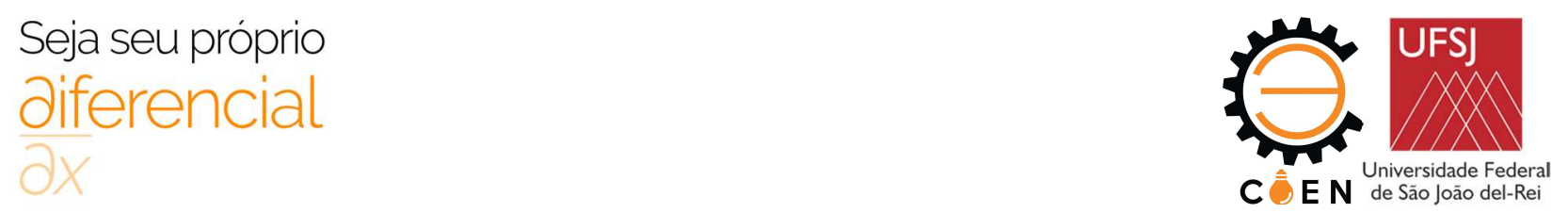

é referente à densidade do espaço vazio dentro da parede externa do objeto. É medido em \% ao invés de mm como é a altura da camada. Se um objeto é impresso com $100 \%$ de preenchimento, ele será completamente sólido por dentro. Quanto maior for a porcentagem de preenchimento, mais resistente e mais pesado o objeto ficará, além de demandar mais tempo e material para que o preenchimento seja feito (ALL3DP, 2016).

\subsection{Processo de impressão e solução de possíveis problemas}

Quanto aos parâmetros que interferem diretamente na impressão temos a retração, que é o parâmetro que diz à impressora para retrair o filamento do bico extrusor para que o filamento pare de sair quando há descontinuidade na peça. Normalmente está sempre ativado, a não ser que a impressão não contenha descontinuidades (ALL3DP, 2016).

A velocidade de impressão está relacionada à velocidade que o bico extrusor vai se movimentar enquanto deposita o filamento. A melhor configuração depende de qual objeto será feito, o material utilizado, a capacidade da impressora e a altura da camada. Para impressões detalhadas é recomendado usar baixas velocidades de impressão (ALL3DP, 2016).

Suportes são estruturas que auxiliam no apoio de objetos $3 \mathrm{D}$ que não possuem material suficiente na base para mantê-los erguidos durante a impressão. Pelo fato dos objetos serem impressos em camadas, partes do objeto que ultrapassem ângulos de 45 graus não terão nada para se apoiarem. Essas são as chamadas saliências (overhangs, tradução nossa) que podem dar uma aparência de borda caída na peça sem os suportes (ALL3DP, 2016).

Tipo de adesão à plataforma é a configuração que afeta o modo como seu modelo vai grudar na mesa de impressão. Há duas configurações principais para ajudar na aderência do modelo na mesa de impressão:

Raft: uma grade horizontal que vai debaixo da peça agindo como uma plataforma que gruda na mesa durante a impressão. Também são muito úteis ao imprimir partes pequenas na base de um modelo 3D. Essa configuração deixa a base da peça mais rugosa;

- $\quad$ Brim: parecido com uma aba de chapéu, são linhas ao redor da base da impressão cuja função é manter as bordas da impressão fixas na base deixando menos marcas. É a indicado como melhor opção para manter o objeto grudado na mesa de impressão (ALL3DP, 2016).

Além dos parâmetros de impressão é importante regular corretamente a impressora e fazer uma checagem periodicamente. A calibração ajuda a assegurar que cada impressão sairá exatamente a mesma, independentemente do equipamento usado para fazê-la. Nada será impresso assimetricamente e a impressão será idêntica sempre (EVANS, 2019).

Limpar a mesa de impressão ajuda a manter as peças grudadas nela. marcas de digitais e restos de impressões passadas podem fazer com que a impressão não grude corretamente. Limpe a mesa regularmente utilizando pano sem fiapos e álcool (GRIESER, 2015).

Nivelar a mesa de impressão é crucial para o sucesso da impressão. Uma mesa bem nivelada é pré requisito para que a primeira camada de impressão seja impressa corretamente. Má nivelamento da mesa pode entortar as primeiras camadas da peça (warping, tradução nossa) e o que resulta no desprendimento da peça da mesa. Nivelar a mesa significa fazer com que o bico extrusor esteja sempre paralelo à mesa (GRIESER, 2015).

A distância entre o bico extrusor e a mesa de impressão afeta diretamente a primeira camada de impressão. Se o bico extrusor está muito afastado da mesa, a peça pode descolar causando o 

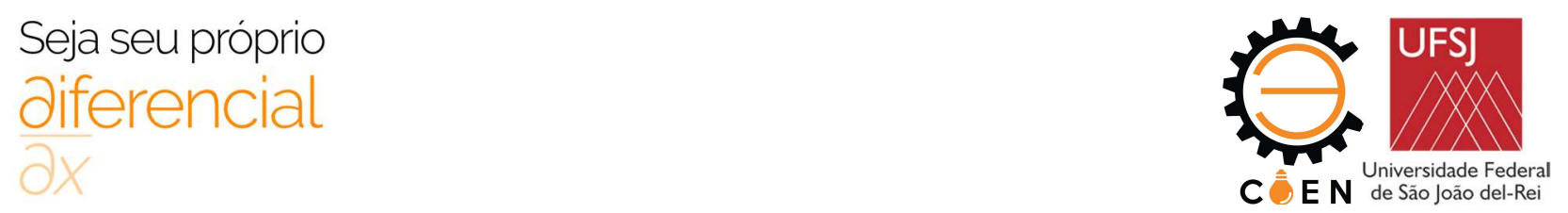

entortamento ou perda da peça, e se está muito próximo da mesa isso pode impedir a saída de material da impressora causando entupimento do bico (GRIESER, 2015).

Alguns dos problemas de impressão ocorrem quando as primeiras camadas de impressão não estão adequadamente fixadas à mesa de impressão. Para melhorar a adesão do filamento, é recomendado cobrir a mesa com fita térmica (normalmente para ABS pois suporta altas temperaturas), ou aplicar cola em bastão ou spray de cabelo (diretamente na mesa ou na fita) (GRIESER, 2015).

O multiplicador de extrusão (extrusion multiplier) é um parâmetro muito útil para ajustar a extrusão de material. Isso auxilia a resolver problemas relacionados à quantidade de material extrudido, relacionado a problemas como: excesso de extrusão (over-extrusion), baixa extrusão (under-extrusion), espaços entre as camadas finais (gaps in top layers), entre outros (SIMPLIFY3D, 2019, tradução nossa).

Velocidades de impressão acima das recomendadas podem gerar problemas como: preenchimento fraco (weak infill), espaços entre o preenchimento e linha externa (gaps between infill and outline), salto entre camadas (layer shifting), dentre outros. Diminuir a velocidade de impressão pode proporcionar melhores resultados finais (SIMPLIFY3D, 2019, tradução nossa).

A temperatura de extrusão é um fator que afeta diretamente o objeto impresso. Caso a temperatura esteja abaixo do recomendado para o material pode acarretar problemas como: separação e divisão entre camadas (layer separation and splitting), entortamento (warping) ou não extrusão no começo da impressão. Quando a temperatura está acima do recomendado, pode gerar: aparecimento de fios ou derretimento (stringing or oozing), aquecimento excessivo (overheating), bico extrusor entupido (clogged extruder), baixa qualidade de pontes (poor bridging), dobras ou quinas grossas (curling or rough corners), dentre outros. A regulagem da temperatura de extrusão está diretamente ligada à qualidade da impressão 3D (SIMPLIFY3D, 2019, tradução nossa).

\subsection{Acabamento}

Para se retirar a peça da superfície de impressão pode ser necessário o uso de uma espátula que ajude a descolar a mesma. Além disso, caso suportes tenham sido necessários, os mesmos devem ser removidos (OROPALLO \& PIEGL, 2015). Para corrigir eventuais erros, é preciso aumentar a precisão de algumas geometrias, suavizar as superfícies obtidas ou remover marcas de suportes. Pode ser necessário fazer um pós-processamento em um processo complementar (FERNANDEZVICENTE et al., 2015). Os procedimentos mais comuns para o acabamento são lixamento e polimento manual ou por jateamento de abrasivo, usinagem tradicional, como furos, ou a usinagem química pela aplicação de um solvente, como a acetona para impressões em ABS (OROPALLO \& PIEGL, 2015). Por fim, o conjunto impresso deve ser montado, ou colado caso a peça tenha sido dividida para ser fabricada em etapas, e, para questões estéticas, o modelo pode ser pintado. Ocorrendo qualquer erro na fabricação, o material pode ser reciclado para fabricar mais material para impressão (AGUIAR, 2016).

\section{CONCLUSÃO}

Para facilitar o entendimento do artigo, a conclusão foi dividida em tópicos:

1. Escolhendo a impressora: 


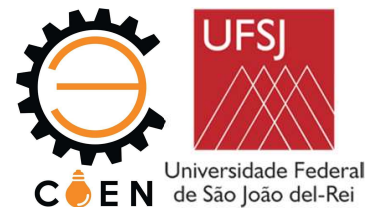

a. usuários novos, usar impressoras de estrutura de arquitetura fechada, mas arquitetura de operação aberta;

b. após adquirir experiência, utilizar uma impressora de arquitetura completamente aberta.

2. Projeto e modelo:

a. verificar as limitações da impressora no manual fornecido ou comunidade;

b. verificar os materiais disponíveis e suas resistências segundo manual da impressora e avaliação do material por outros usuários;

c. elaborar a geometria do objeto que será impresso ou, como alternativa, escanear o objeto ou até baixar em repositórios;

d. fazer simulação, se possível;

e. definir parâmetros de impressão:

i. altura da camada, definindo a qualidade da impressão e o tempo, definida pelo diâmetro do bico extrusor, o mais comum é $0.2 \mathrm{~mm}$ para qualidade mediana, $0.3 \mathrm{~mm}$ e acima para qualidade grosseira e $0.1 \mathrm{e}$ abaixo para qualidade fina ;

ii. espessura da parede externa, manter a quantidade necessária para garantir a resistência mecânica da peça, sendo recomendado manter o mínimo de 2 camadas ou $0.8 \mathrm{~mm}$ de espessura, configuração padrão;

iii. densidade de preenchimento, sendo o ideal, utilizando o preenchimento de 10$20 \%$ para peças normais e $75-100 \%$ para maior resistência;

3. Impressão e erros:

a. parâmetros de impressão (que influenciam diretamente no processo de impressão):

i. retração, para evitar excesso de material durante pausas ou movimentos entre faces, fazendo parte da configuração padrão do fatiador, normalmente $1 \mathrm{~mm}$;

ii. velocidade de impressão, mantê-la moderada para melhor qualidade de impressão, sendo $60 \mathrm{~mm} / \mathrm{s}$ como configuração padrão mas podendo chegar a $40 \mathrm{~mm} / \mathrm{s}$ para melhorias na qualidade superficial da peça;

iii. suporte, varia de acordo com a capacidade da impressora, geometria da peça e material utilizado, normalmente configurado para um ângulo de 50 graus ou menos;

iv. tipo de adesão à plataforma de impressão, tem influência principalmente na primeira camada de impressão, assegurando também que a peça permaneça fixada à mesa durante todo o processo;

b. calibração, sempre necessário checar antes de começar o processo de impressão pois pode acarretar vários problemas posteriores:

i. nivelamento da mesa de impressão, para que a peça se fixe corretamente sem partes mais folgadas ou descolamento entre camadas;

ii. tensionamento das correias e eixos, pode evitar vibrações na superfície da peça assim como saltos entre as camadas;

iii. calibração dos motores, para que não haja erro na deposição de materiais entre as camadas;

c. limpeza da superfície de impressão para melhor aderência da peça;

IX COEN - Congresso de Engenharias da UFSJ

Interconexão. 
d. temperatura de impressão varia dependendo do material utilizado e da temperatura no local, podendo ser necessário enclausuramento da impressora para controle da temperatura durante a impressão e mesa de impressão aquecida dependendo do material.

i. o enclausuramento da máquina pode influenciar na qualidade final da peça principalmente de peças feitas em ABS devido à sua forte contração ao esfriar, o enclausuramento ajuda a manter a temperatura de impressão constante durante a impressão, às vezes é necessário pré-aquecer a câmara antes de dar início à impressão;

ii. mesa de impressão aquecida é indicada principalmente para peças em ABS devido ao entortamento (warping) causado pelas contrações do material,

podendo também ser utilizada para auxiliar na fixação da peça na mesa independente do material.

4. Acabamento:

a. remover da superfície, caso necessário utilize uma espátula, com cuidado para não danificar as superfícies da peça e da impressora;

b. Remover os suportes, com cuidado para não danificar a peça, para algumas impressoras o suporte pode ser solúvel em água ou outro solvente específico;

c. lixar as imperfeições na peça, atentando-se para não remover material em excesso;

d. fazer a furação quando necessário, podendo utilizar enxertos para fortalecer a peça e fazer rosca;

e. aplicar o solvente para suavização da superfície, com cuidado para não exceder na aplicação direta, ou observar constantemente quando utilizar a submersão da peça no solvente diluído ou em vapor;

f. fazer pintura com tintas compatíveis com os materiais utilizados na impressão.

\subsection{Recomendações}

Para auxílio em soluções de problemas, aprimoramentos de impressoras e construção de uma impressora própria, os autores recomendam as Wikis listadas abaixo e seus Fóruns. Nesses sites há projetos de impressora completos, inclusive a parte elétrica e a programação necessária, além de recomendações quanto ao uso da impressora, para solução de erros e calibração das máquinas. Todo conteúdo é criado por uma comunidade aberta.

- RepRap - projeto comunitário internacional para o desenvolvimento colaborativo das impressoras 3D de arquitetura aberta (REPRAP, 2019);

- Appropedia - comunidade em inglês para desenvolvimento e compartilhamento de soluções colaborativas (APPROPEDIA, 2019, tradução nossa).

Além disso, também recomendamos os canais na plataforma de vídeo Youtube, que apresentam dicas, projeto e recomendações sobre impressão 3D:

- Ricardo Cavallini - canal em português do fundador de uma plataforma de educação e inovação focada em prototipagem e desenvolvimento de produtos utilizando a

IX COEN - Congresso de Engenharias da UFSJ Interconexão. 


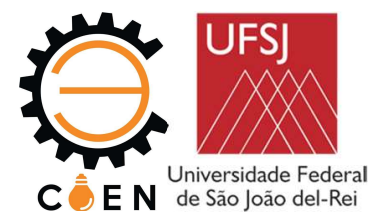

impressora 3D (CAVALLINI, 2019);

- Thomas Sanladerer - canal em inglês com guias, tutoriais, dicas e avaliações de impressoras 3D (SLANDERER, 2019, tradução nossa);

- Maker Muse - canal em inglês de revisão, avaliação e projetos de impressão 3D (DEVESON, 2019, tradução nossa);

- CNC Kitchen - canal em inglês sobre Impressoras 3D, CNC, CAD, Laser, Arduino e outros projetos complementares (HERMAN, 2019, tradução nossa);

- 3D Printing Nerd - canal em inglês com o objetivo de educar, informar e até advogar em nome da comunidade " 3 D printing” (TELLING, 2019, tradução nossa).

Outros sites relevantes em relação à dicas de impressão e solução de problemas são:

- 3D Beginners - site em inglês dedicado à impressão 3D para iniciantes (EVANS, 2019, tradução nossa);

- $3 D$ Lab - Blog de um dos principais fornecedores de suprimentos para impressão 3D no Brasil, apresentam dicas e recomendações para o uso dos equipamentos e suprimentos (3DLAB, 2019);

- $A L L 3 D P$ - site e revista em inglês fornecedora de material educativo e de entretenimento sobre impressão 3D para iniciantes e profissionais (ALL3DP, 2019, tradução nossa);

- Simplify $3 D$ - Blog do fornecedor do software simplify $3 D$, com dicas de impressão e resolução de problemas (SIMPLIFY3D, 2019, tradução nossa).

\section{AGRADECIMENTOS}

Os autores agradecem o apoio da FAPEMIG, do CNPq e da CAPES na concessão de cotas de bolsas e financiamentos para os diversos Programas Institucionais e laboratórios que colaboraram com a presente pesquisa. Agradecem também aos graduandos, auxiliares técnicos e discentes da universidade integrantes dos mesmos.

\section{DIREITOS AUTORAIS}

Os autores são os únicos responsáveis pelo conteúdo das informações contidas neste artigo.

\section{REFERÊNCIAS}

3DLAB. Blog. 2019. Disponível em: <https://3dlab.com.br/blog/>. Acesso em: 14 de jul. de 2019.

AGUIAR, Leonardo De Conti Dias. Um processo para utilizar a tecnologia de impressão $3 \mathrm{~d}$ na construção de instrumentos didáticos para o ensino de ciências. 2016.

ALL3DP. 3D Slicer Settings for Beginners -8 Things You Need to Know. 2019. Disponível em: $<$ https://m.all3dp.com/3d-slicer-settings-beginners-8-things-need-know/>. Acesso em: 14 de Jul. De 2019.

APPROPEDIA. Appropedia Wiki. 2019. Disponível em: < https://www.appropedia.org/>. Acesso em: 10 de jul. de 2019.

IX COEN - Congresso de Engenharias da UFSJ Interconexão. 


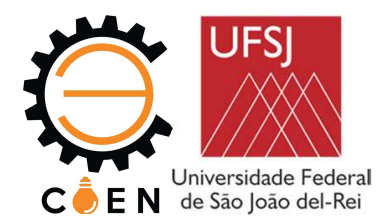

AUTODESK Inc. Softwares educacionais gratuitos para alunos, professores e instituições educacionais que utilizam produtos Autodesk. 2019. Disponível em: $<$ https://www.autodesk. com.br/education/free-educational-software $>$. Acesso em: 14 de jul. de 2019.

BAUMANN, Felix; ROLLER, Dieter. Vision based error detection for 3D printing processes. In: MATEC web of conferences. EDP Sciences, 2016. p. 06003.

BERMAN, Barry. 3-D printing: The new industrial revolution. Business horizons, v. 55, n. 2, p.155$162,2012$.

CAVALLINI, Ricardo. RicardoCavallini. 2019. Disponível em: $<$ https://www. youtube.com/user/ricardocavallini/about>. Acesso em: 10 de jul. de 2019.

CULMONE, Costanza; SMIT, Gerwin; BREEDVELD, Paul. Additive manufacturing of medical instruments: A state-of-the-art review. Additive Manufacturing, 2019.

CULTS. Cults. 2019. Disponível em: <https://cults3d.com/>. Acesso em: 10 de jul. de 2019. DEVESON, Angus. Maker's Muse. 2019. Disponível em: <https://www.youtube.com/ user/TheMakersMuse/about>. Acesso em: 10 de jul. de 2019.

EVANS, Justin. How to Calibrate A 3D printer. 2019. Disponível em: <https://www.3dbeginners.com/how-to-calibrate-a-3d-printer/>. Acesso em: 14 de Jul. de 2019.

FERNANDEZ-VICENTE, Miguel; CANYADA, Miquel; CONEJERO, Andres. Identifying limitations for design for manufacturing with desktop FFF 3D printers. International Journal of Rapid Manufacturing, v. 5, n. 1, p. 116-128, 2015.

GRIESER, Franz. 3D Printing Quality Issues: 10 Tricks To Avoid Them. 2015. Disponível em $<$ https://all3dp.com/3d-printing-quality/>. Acesso em: 14 de Jul. de 2019.

HERMANN, Stephan. CNC Kitchen. 2019. Disponível em: $<$ https://www.youtube.com/channel/UCiczXOhGpvoQGhOL16EZiTg/about>. Acesso em: 10 de jul. de 2019.

HIEMENZ, Joe. 3D printing with FDM: How it Works. Stratasys Inc, v. 1, p. 1-5, 2011. KAUARK, Fabiana da Silva; MANHÃES, Fernanda Castro; MEDEIROS, Carlos Henrique. Metodologia da pesquisa: um guia prático. 2010.

KUN, Krisztián. Reconstruction and development of a 3D printer using FDM technology. Procedia Engineering, v. 149, p. 203-211, 2016.

MAKERBOT Industries. Thingverse. 2019. Disponível em: $<$ https://www.thingiverse.com/>. Acesso em: 10 de jul. de 2019.

MARQUARDT, Tahnee; ZHENG, Emmi. History of 3D printing. 2016.

MYMINIFACTORY. MyMiniFactory. 2019. Disponível em: <https://www. myminifactory.com/>. Acesso em: 10 de jul. de 2019.

OROPALLO, William; PIEGL, Les A. Ten challenges in 3D printing. Engineering with Computers, v. 32, n. 1, p. 135-148, 2016.

REPABLES. Repables. 2019.Disponível em: < https://repables.com/>. Acesso em: 10 de jul. de 2019. jul. de 2019.

REPRAP. RepRap/pt. 2019. Disponível em: <http://reprap.org/wiki/RepRap/pt>. Acesso em: 10 de

REPRAP. Print Troubleshooting Pictorial Guide. 2018. Disponível em: $<$ https://reprap.org/wiki/Print_Troubleshooting_Pictorial_Guide>. Acesso em: 10 de jul. de 2019.

IX COEN - Congresso de Engenharias da UFSJ Interconexão. 
SANLANDERER, Thomas. ThomasSanladerer. 2019. Disponível em: $<$ https://www.youtube.com/user/ThomasSanladerer/about $>$. Acesso em: 10 de jul. de 2019.

SIMPLIFY3D. Print Quality Troubleshooting Guide. 2019. Disponível em $<$ https://www.simplify3d.com/support/print-quality-troubleshooting/>. Acesso em: 14 de Jul. de 2019.

SONG, Ruoyu; TELENKO, Cassandra. Material and energy loss due to human and machine error in commercial FDM printers. Journal of cleaner production, v. 148, p. 895-904, 2017.

STRATASYS. GrabCAD Community Library.2019. Disponível em: $<$ https://grabcad.com/library>. Acesso em: 10 de jul. de 2019.

TELLING, Joel. 3D PrintingNerd. 2019. Disponível em: <https://www. youtube.com/channel/UC_7aK9PpYTqt08ERh1MewlQ/about>. Acesso em: 10 de jul. de 2019.

\section{D PRINTING: A PRACTICAL GUIDE}

Pedro Coelho Silva ${ }^{(1)}$ (ocoelhopedro@gmail.com), Rafael Souza Santandrea ${ }^{(1)}($ r.santandrea97@gmail.com), Victor Lattaro Volpini ${ }^{(1)}$ (victorlattaro@gmail.com), Lincoln Cardoso Brandão ${ }^{(1)}$ (lincoln@ufsj.edu.br)

(1) Universidade Federal de São João del-Rei (UFSJ) - Engenharia Mecânica - Praça Frei Orlando, n 170 - Centro - São João delRei-MG

ABSTRACT: $3 D$ Printing is an versatile technology with potential to start major changes in how we manufacture products. Also called Additive Manufacturing, had its main methodologies created in the 80's. Only after the expiration of the main patents its popularization started. The machine operation is still considered complex for beginner users, mainly due to the lack of documentation. Using a methodology with the objective to identify in literature and the practical experience of the authors the minimum necessary procedures to use this kind of printer. This article also presents the results in a simple and objective way, being a practical guide for new users. It contains recommendations for purchasing a $3 D$ printer and the softwares needed for its operation. Also include the settings that can be available and how it interferes with the final quality. Finally how to avoid printing errors and the finishing process to the printed object. In conclusion, an objective list will comment each of the topics, with recommendations by the authors.

KEYWORDS: $3 d$ printing, guide, additive manufacturing. 